\title{
COVID-19 aus Sicht der Infektiologie
}

Elham Khatamzas, Camilla Rothe, Inge Kroidl

\section{Die SARS-CoV-2-Pandemie stellt die Welt vor beispiellose Herausforderungen. Die besonderen Aspekte der rapiden Ausbreitung des Erregers sowie der Mangel an spezifischen prophylaktischen und therapeutischen Maßnahmen erfordern infek- tiologische Expertise und interdisziplinäre Zusammenarbeit auf allen Ebenen.}

\section{Epidemiologie}

Im Dezember 2019 gab es die ersten Fallberichte einer atypischen Pneumonie unklarer Genese aus der Region um Wuhan in China. Am 7. Januar 2020 wurde der Erreger SARS-CoV-2 (SARS: Severe Acute Respiratory Syndrome), ein neuartiges Coronavirus, als Ursache für die Erkrankung erkannt [1]. Der Ausbruch dieser v. a. respiratorischen Erkrankung, Coronavirus Disease 2019 (COVID-19), wurde nach rapider globaler Ausbreitung am 12. März 2020 von der Weltgesundheitsorganisation zur Pandemie erklärt. Die Anzahl bisher bestätigter Fälle weltweit beträgt bisher mehr als 4,7 Millionen mit mehr als 300000 Todesfällen (Stand 18. Mai 2020, John Hopkins COVID-19 Map).

\section{Virologie}

SARS-CoV-2 gehört zum Genus der Beta-Coronaviren. Humane Coronaviren verursachen meist leicht verlaufende akute respiratorische Erkrankungen. Allerdings haben im 21. Jahrhundert bereits 2 neuartige Coronaviren zu Epidemien mit schwerwiegenden akuten respiratorischen Infektionen und hoher Letalität geführt: Im Jahr 2002 SARS-CoV aus Südchina und im Jahr 2012 Middle East Respiratory Coronavirus (MERS-CoV) mit Ursprung in Saudi-Arabien. Bei beiden Erkrankungen handelt es sich um Zoonosen. Das Genom von SARS-CoV-2 zeigt eine 21 \%ige Differenz zu SARS$\mathrm{CoV}$ auf. Auch bei SARS-CoV-2 weisen genetische Analysen auf Fledermäuse als das natürliche Reservoir von Coronaviren und andere Tiere als potenzielle Zwischenwirte hin [2].

Coronaviren sind behüllte RNA-Viren mit dem derzeit größten bekannten RNA-Genom. Sie weisen nach außen ragende Proteinstrukturen auf, die elektronenmikroskopisch an das Bild einer Krone (lateinisch corona) erinnern. Für die Pathogenese und als Ansatzpunkte für diagnostische Verfahren sowie potenzielle Therapeutika und Impfstoffe sind die vom Virusgenom kodierten viralen Proteine wichtig. Hierzu gehört das auf der Virushülle exprimierte Spike-Protein (S), das durch eine Rezeptorbindungsdomäne (RBD) das humane Angiotensin-Converting-Enzym-2-Molekül (hACE2-Molekül) als Rezeptor usurpiert. ACE2 wird unter anderem von alveolären und intestinalen Epithelzellen exprimiert. Nach der Bindung wird das S-Protein von einer auf den Membranen der Wirtszellen vorhandenen Serinprotease, TMPRSS2 (transmembrane protease serine 2), gespalten. Dies wiederum ermöglicht die Fusion mit der Wirtsmembran und somit den Eintritt des Virus in die Zielzelle [3].

\section{Transmission}

Kaum ein für SARS-CoV-2 relevantes Thema hat solch kontroverse Diskussionen ausgelöst wie die Frage der Übertragungswege. Zweifellos erfolgt die Übertragung hauptsächlich über Tröpfchen. Infizierte sind bereits vor Beginn einer typischen Symptomatik infektiös und können so unbemerkt das Virus verbreiten [4, 5]. Experimentelle Studien, in denen mit SARS-CoV-2 angereicherte Aerosole, d. h. Partikel mit einem Durchmesser $<5 \mu \mathrm{m}$, unter verschiedenen Bedingungen untersucht wurden, zeigten, wie es zu einer Verteilung der Viren kommen kann. Diese Studien weisen darauf hin, dass es potenziell zu einer Übertragung durch diese Mikrotröpfchen kommen kann, und zwar v. a. dann, wenn kein Schutz durch eine Mund-Nasen-Maske vorhanden ist [6]. Aufgrund des Nachweises von SARS-CoV-2 auf unterschiedlichen Oberflächen ist derzeitig auch von einer möglichen Kontaktübertragung auszugehen [7].

\section{Klinik}

Die ersten größeren Fallserien aus China enthielten v. a. Berichte von Patienten mit schweren Pneumonien [8]. In weiteren Untersuchungen wurde gezeigt, dass ungefähr 80 \% der Fälle mild verlaufen, mit auch einem erheblichen Anteil asymptomatischer bis oligosymptomatischer Personen. Allerdings benötigen bis zu $20 \%$ der Patienten eine stationäre, darunter $6 \%$ eine intensivmedizinische Versorgung. In Italien betrug der Anteil an Patienten, die intensivmedizinisch versorgt werden mussten, bis zu $12 \%$. Die klinischen Verläufe sind u. a. abhängig von den lokalen demografischen Verhältnissen.

Die Erkrankung manifestiert sich als eine Infektion der Atemwege, die v. a. mit Fieber und nichtproduktivem 


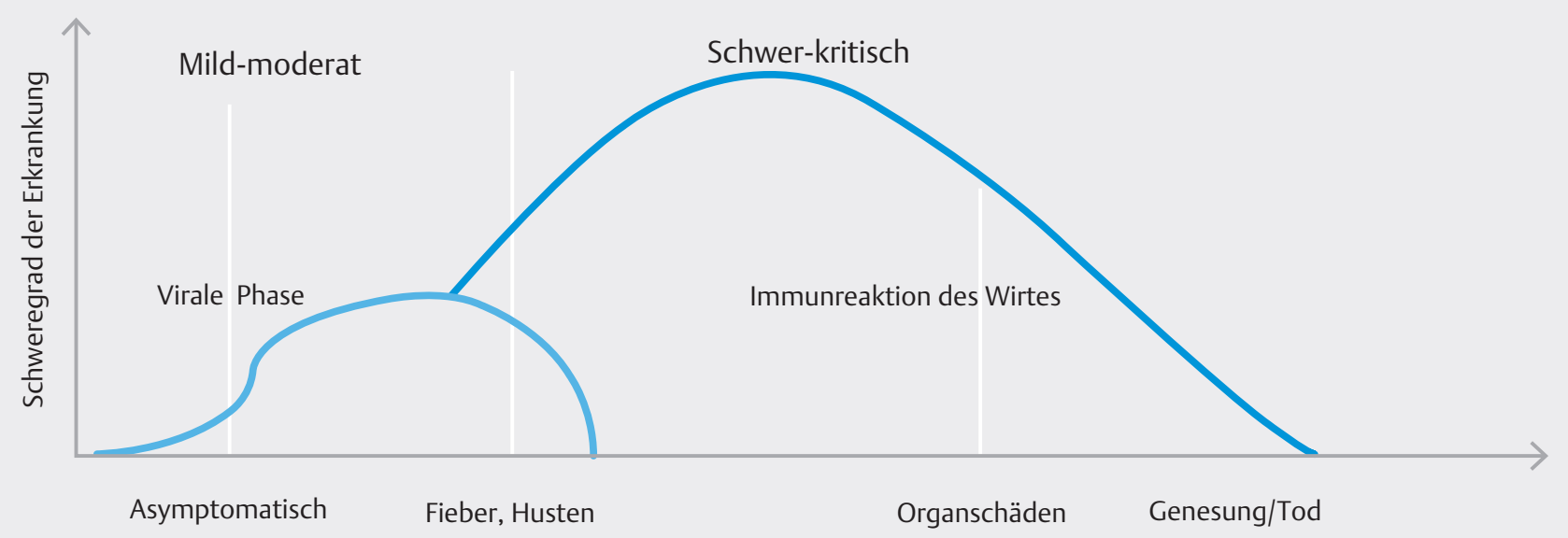

- Abb. 1 Schema des COVID-19-Krankheitsverlaufs.

Husten einhergeht. Weitere Symptome umfassen eine teilweise ausgeprägte Abgeschlagenheit, Myalgien, Kopfschmerzen sowie Verlust des Geruchs-/Geschmackssinns. Letzteres wird sogar, wenn vorhanden, als besonders charakteristisches Symptom einer COVID-19Erkrankung erkannt. Die Oxygenierung, z. B. mittels Überwachung der peripheren Sauerstoffsättigung sowie der Atemfrequenz, dient als wichtiges Maß zur angemessenen Stratifizierung der Patienten. Die sogenannte „stille Hypoxie“ der Erkrankten ist hier besonders hervorzuheben. Dabei sind Patienten trotz manifester Hypoxämie relativ symptomarm und es kann bei peripheren Sauerstoffsättigungen von $<90 \%$ zu einer sehr rapiden respiratorischen Verschlechterung kommen. Diese Patienten erfordern eine engmaschige stationäre Überwachung.

\section{Risikogruppen}

Alle bisher veröffentlichten internationalen Registeranalysen zeigen, dass das Vorliegen von Herz- und Kreislauferkrankungen, v. a. Hypertonus, sowie Diabetes mellitus und Übergewicht mit erhöhtem Risiko einer schweren Erkrankung nach Infektion mit SARS-CoV-2 einhergeht. Obwohl jede Altersgruppe betroffen sein kann, erkranken Patientengruppen höheren Alters signifikant häufiger schwer. Weiterhin gibt es Hinweise darauf, dass männliche Patienten schwerer erkranken als weibliche [8]. Außer kleineren Fallberichten gibt es bisher kaum Daten hinsichtlich immunsupprimierter Patientenpopulationen und des Einflusses einer Immunsuppression auf den Krankheitsverlauf.

\section{Pathogenese}

Gegenwärtig wird der klinische Krankheitsverlauf in 2 Stadien unterteilt ( $\triangleright$ Abb. 1). Ein Initialstadium mit vira- ler Replikation über mehrere Tage mit Aktivierung des angeborenen Immunsystems zeigt sich mit klinisch milder Symptomatik. Anschließend wird die adaptive Immunantwort ausgelöst und die Viruslast reduziert. Diese Reaktion kann zu einer überschießenden inflammatorischen Reaktion mit starkem Anstieg von Zytokinen wie IL-6 und IL-1RA führen, einem sogenannten Zytokinsturm [9]. Trotz der v. a. im Lungenparenchym auffälligen Schädigung ist von einer pathologischen generalisierten Entzündung auszugehen. In einer Autopsie-Studie konnte virale RNA in Zellen zahlreicher Organe nachgewiesen werden, u. a. auch in Niere, Leber und Herz [10].

\section{Diagnostik}

\section{Direkter Erregernachweis von SARS-CoV-2-RNA}

Der Goldstandard zur Diagnose einer neuen Infektion ist der Nachweis von SARS-CoV-2-Nukleinsäuren. Dies erfolgt nach entsprechender Probenaufbereitung, Extraktion der Nukleinsäuren und anschließender ReverseTranskriptase-PCR-spezifischer Zielsequenzen des Genoms. Hier gibt es bereits mehrere kommerzielle Testsysteme. Verwendet wird hierfür Material, das aus den oberen und unteren Atemwegen, z. B. mit einem nasopharyngealen Abstrich, gewonnen wird. Trotz negativer Initialdiagnostik sollte bei weiterhin bestehendem klinischem und/oder radiologischem Verdacht eine fortführende Diagnostik mit Wiederholung der Tests durchgeführt werden. Dies ist v. a. wichtig bei stationären Patienten, um eine mögliche nosokomiale Weiterverbreitung zu vermeiden. Generell war und bleibt die größte Herausforderung hierbei allerdings weiterhin der steigende Bedarf an zeitnaher Testung und Analyse einer größeren Anzahl an Personen bei noch limitierter Testkapazität. 


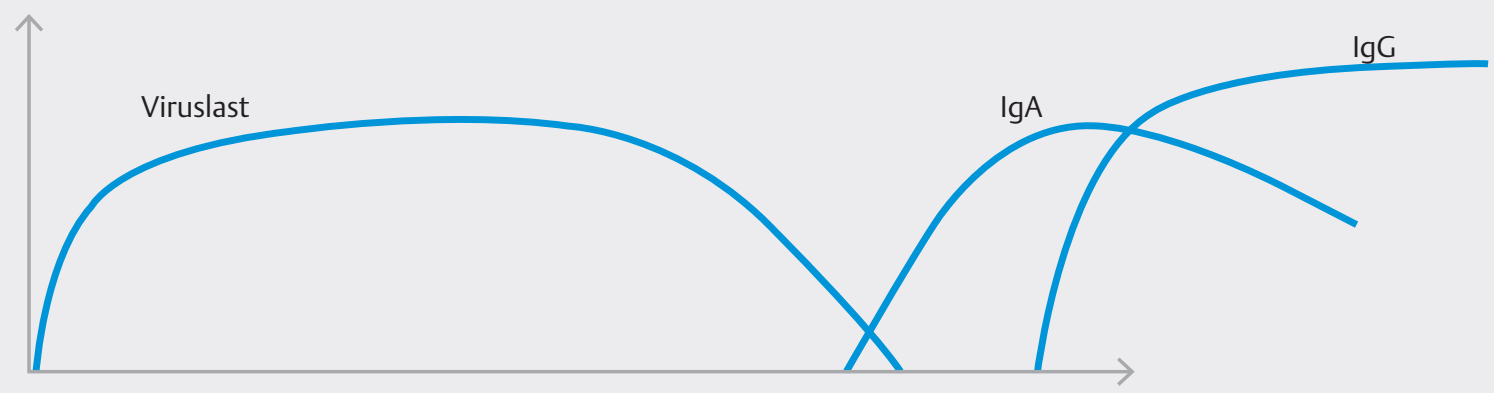

Zeit in Tagen

- Abb. 2 Schema der SARS-CoV-2-Antikörperantwort.

\section{Verlängerter Nachweis von SARS-CoV-2-RNA}

Gelegentlich lassen sich bei einer Gruppe von genesenen Patienten nach durchgemachter Infektion in den Atemwegen sowie im Stuhl wiederholt noch virusspezifische Nukleinsäuren nachweisen. Beschrieben ist eine Zeitdauer von bis zu 60 Tagen nach Symptombeginn [11]. Bisher gibt es keine klinischen oder epidemiologischen Hinweise auf eine potenzielle persistierende Infektiosität. Detaillierte virologische und immunologische Untersuchungen an der ersten Kohorte in Deutschland weisen darauf hin, dass es sich hierbei lediglich um RNA-Fragmente und nicht um infektiöses, replizierenden Virus handeln könnte [12]. Auch bei anderen Viren, wie Masern, Ebola oder Zika, lässt sich nach durchgemachter Infektion virale RNA bis zu 6 Monate später detektieren [13].

\section{Nachweis von SARS-CoV-2-spezifischen Antikörpern}

Der Nachweis von SARS-CoV-2-spezifischen Antikörpern kann zur Bestätigung einer durchgemachten Infektion und letztendlich Abschätzung der Seroprävalenz in bestimmten Kohorten sowie der allgemeinen Bevölkerung dienen. Verschiedene kommerzielle Anbieter bieten inzwischen Antikörpertests für SARS-CoV-2 an. Der ELISA der Firma Euroimmun AG (Lübeck) weist IgG spezifisch für die S1-Domäne des Spike-Proteins von SARS-CoV-2 sowie relevante Teile der bereits erwähnten Rezeptorbindungsdomäne (RDB) nach und hat eine Spezifität von 99,1\% und eine Sensitivität von 90,9\%. Für SARS-CoV-2IgA derselben Firma wird eine $100 \%$ ige Sensibilität und 88 \%ige Spezifität angegeben.

Diese IgG/IgA-ELISA wurden in einer bereits publizierten Untersuchung sowie weiteren in Deutschland stattfindenden Studien benutzt $[14,15]$. Seit Anfang Mai bietet die Fa. Roche (Penzberg, Deutschland) einen weiteren ELISA (Elecsys ${ }^{\circledR}$ Anti-SARS-CoV-2-Immunoassay) an, der eine Sensibilität von $100 \%$ und Spezifität von $99,8 \%$ verspricht und in die laufenden Studien integriert werden wird.
Wichtig bei der Verwendung und Beurteilung von Antikörpertests für SARS-CoV-2 ist auch der Zeitpunkt der Untersuchung: Nach einer Infektion mit SARS-CoV-2 kommt es ca. 1-3 Wochen später zur nachweisbaren Produktion von IgA-Antikörpern, die typischerweise bei Infektionen der Schleimhäute gebildet werden. Etwas später erst können IgG-Antikörper nachgewiesen werden ( Abb. 2). So kann die Sensibilität eines ELISA-Tests zu niedrig angegeben werden, wenn die Untersuchung zu einem zu frühen Zeitpunkt stattgefunden hat.

Nachdem erste Berichte über COVID-19 hauptsächlich auf Patienten im Krankenhaus mit schwerem Verlauf fokussiert waren, gibt es inzwischen Berichte von oligosymptomatischen SARS-CoV-2-Infizierten. Dabei wurden Patienten beschrieben, die trotz gesichertem PCR-Nachweis von SARS-CoV-2 keine Antikörper gegen das Virus gebildet hatten. Ob bei diesen Patienten ein anderer Weg der Immunantwort vorrangig aktiv war und z. B. eine T-Zell-vermittelte Immunität ausgebildet wurde, ist Teil laufender Studien. Oligosymptomatische Patienten scheinen im Vergleich mit hospitalisierten Patienten erst Tage bzw. Wochen später Antikörper zu produzieren. Der Schweregrad einer COVID-19-Erkrankung war in einer Studie assoziiert mit der Höhe der IgG-Antikörper gegen SARS-CoV-2 [16].

Merke

Momentan werden Antikörpertests vorrangig durchgeführt, um zu überprüfen, ob es in der Vergangenheit zu einer SARS-CoV-2-Infektion gekommen ist. Aussagen zur Immunität gegen SARS-CoV-2, gerne gesehen als Freibrief für risikoreiche Tätigkeiten, lassen sich daraus nicht ableiten.

Zur Frage, ob die Antikörper gegen eine erneute SARSCoV-2-Infektion schützen, werden Neutralisationstests durchgeführt, in denen Plasma von Rekonvaleszenten mit Virus inkubiert und dann die Infektiosität dieser Mischung auf Zellen getestet wird. Erste, noch nicht publizierte Ergebnisse der laufenden Studien weisen darauf hin, dass es Patienten mit nachgewiesener SARS-CoV-2- 
Infektion und messbaren spezifischen IgG-Antikörpern gibt, denen die Fähigkeit zur Neutralisation fehlt [14]. Aber selbst wenn neutralisationsfähige IgG-Antikörper dokumentiert werden konnten, kann über die Dauer dieses potenziellen Immunschutzes derzeit nur spekuliert werden. Verlaufsuntersuchungen von Patienten nach Infektion, sogenannte COVID-19-Genesene, könnten in Zukunft auf nationaler und internationaler Ebene hierzu neue Erkenntnisse schaffen,

\section{SARS-CoV-2-unabhängige Erregerdiagnostik}

Zusätzlich zu der standardisierten SARS-CoV-2-Diagnostik sollte bei stationären Patienten abhängig vom klinischen Schweregrad eine weiterführende mikrobiologische Diagnostik durchgeführt werden. Koinfektionen mit alternativen respiratorischen Erregern wie Influenza, RSV und Mykoplasmen sind beschrieben. Entsprechende Untersuchungen von Blut und respiratorischem Material sollten hier zum Basisprogramm gehören.

Bei bestimmten Risikogruppen, v. a. solchen unter intensivmedizinischer Behandlung, sollten auch invasive Pilzinfektionen als nosokomial erworbene Infektionen erwogen und mit entsprechender Diagnostik abgeklärt werden [17]. Weiterhin ist eine differenzierte erweiterte Erregerdiagnostik bei allen immunsupprimierten Patienten mit COVID-19 zwingend.

\section{Laborparameter}

Besonderheiten bei der Messung von Laborparametern sind eine Erhöhung der inflammatorischen Parameter (CRP, IL-6, Ferritin) sowie das Auftreten einer Lymphopenie und Eosinopenie im peripheren Blutbild. Im klinischen Alltag ist die regelmäßige Überwachung dieser Parameter gegebenenfalls unter Zunahme der LDH sowie der D-Dimere von Bedeutung und könnte zusätzlich zu anderen Scores zur Risikoabschätzung dienen.

\section{Bildgebung}

Interessanterweise lassen sich auch bei Patienten mit milden Verläufen typische Veränderungen in der pulmonalen Bildgebung demonstrieren, bei mindestens $60 \%$ im konventionellen Röntgenbild und bei $85 \%$ in der CTUntersuchung der Lunge. Zu den typischen beschriebenen Veränderungen gehören multifokale, peripher gelegene milchglasartige Infiltrate („ground glass“), meistens bilateral, die im Verlauf der Erkrankung als sogenannte „crazy paving“-Konsolidierung dominieren können (s. unten) [18, 19]. Während diese Veränderungen eine hohe Sensitivität vorweisen, sind sie keineswegs spezifisch für COVID-19. Zunehmend werden auch bettseitige Ultraschalluntersuchungen der Lunge zur Diagnostik und v. a. zur Verlaufskontrolle von fokalen konfluierenden B-Linien durchgeführt. Unsere bisherigen Erfahrungen zeigen, dass diese radiologischen Veränderungen nach durchgemachter Infektion regredient sind.

\section{BILDGEBUNG}

CT-morphologische Veränderungen, die suggestiv

für eine COVID-19-Pneumonie sind*:

- frühe dominante Milchglastrübungen

- späte dominante „crazy paving”-Konsolidierung

- organisierende Pneumonie

- Lokalisation: peripher und posterior

- Verteilungsmuster: bilateral, multifokal

- intraläsional erweiterte Blutgefäße

- keine mediastinale oder hiläre Lymphadenopathie

*bei hoher lokaler Prävalenz und/oder individueller Prä-Test-Wahrscheinlichkeit

\section{Therapie}

\section{Supportive Maßnahmen}

Aufgrund fehlender Evidenz für Substanzen mit direkter SARS-spezifischer Wirksamkeit und der bisher enttäuschenden Berichte über die bisher eingesetzten Substanzen beruht die Behandlung von COVID-19-Patienten v. a. auf den Prinzipien der „Best Standard of Care“ in Kombination mit intensivmedizinischen Maßnahmen. Alle weiteren unten aufgezählten Substanzen sollten unseres Erachtens im Rahmen von klinisch kontrollierten Studien und nur in Einzelfällen auf der Basis eines Off-LabelVersuchs verabreicht werden.

\section{Antimikrobielle Therapie}

Der unsachgemäße Einsatz von Antibiotika führt zu einer stetigen Zunahme von Resistenzen sowie Veränderung des Mikrobioms. Zusammenfassende Analysen zeigen, dass eine antimikrobielle Therapie bei bis zu $72 \%$ der Patienten mit COVID-19 verschrieben wurde [20], obwohl eine bakterielle oder mykotische Koinfektion lediglich bei $8 \%$ der Patienten vorlag. Daten zur Erregerdiagnostik fehlen. In der Studie von Zhou et al. war die Rate an Sekundärinfektionen im Vergleich signifikant höher in der Gruppe mit erhöhter Mortalität trotz einheitlichem Einsatz von Antibiotika in beiden Gruppen [8].

Im klinischen Alltag kann die Bestimmung des Procalcitonins vor Erwägung einer antibiotischen Therapie angepasst an die lokalen Therapieempfehlungen hilfreich sein. Zu erwähnen ist auch der vermehrte Einsatz des Makrolidantibiotikums Azithromycin aufgrund potenzieller SARS-CoV-2-spezifischer Wirksamkeit ähnlich wie das inzwischen sehr kontrovers diskutierte Mittel Hydroxychloroquin; die endgültigen Ergebnisse laufender randomisierter Studien werden hierzu Klarheit bringen [21, 22]. 


\section{Potenzielle Anti-SARS-CoV-2-Therapie}

Erfahrungen aus China zur Bewältigung der lokalen Epidemie haben vielseitige Erfahrungsberichte mit unterschiedlichen Therapieansätzen, aber ohne klare Evidenz erbracht. Mehrere Wirkstoffe zeigen in vitro eine Aktivität gegen SARS-CoV-2 und sind Gegenstand weiterer Untersuchungen. Obwohl die Ergebnisse größerer kontrollierter Studien, wie dem RECOVERY-Trial, noch ausstehen, gibt es vermehrte Berichte über die fehlende Wirksamkeit von Therapeutika wie den HIV-Protease-Inhibitor Lopinavir/Ritonavir sowie Hydroxychloroquin.

Das Nukleosid-Analogon Remdesivir hemmt neben Coronaviren auch andere Viren. Die Ergebnisse einer kontrollierten Studie zur Therapie von Ebola-Fieber zeigten keinen Effekt auf die Mortalität [23]. In einer chinesischen Studie wurde auch kein eindeutiger Vorteil einer Behandlung mit Remdesivir gezeigt. Aktualisierte Daten aus weiteren Studien hierzu werden erwartet.

Weitere derzeit in Prüfung befindliche Ansatzkonzepte basieren auf der Therapie mit immunmodulatorischen Substanzen wie Tocilizumab, einem monoklonalen Antikörper, der spezifisch die Bindung von Interleukin 6 an den Rezeptor hemmt. Auf „machine learning“ basierende Algorithmen zeigten außerdem, dass Inhibitoren der Janus-Kinase (JAK), insbesondere Ruxolitinib, die Endozytose des Virus durch den ACE2-Rezeptor hemmen.

Der Einsatz von Rekonvaleszentenplasma gilt als einer der vielversprechendsten verfolgten Ansätze. Hier wird das Plasma mit Antikörpern von genesenen COVID-19-Patienten zur Behandlung von schweren Verläufen eingesetzt. Einzelne Fallberichte mit jeweils 5 und 10 Patienten zeigen, dass eine einzelne Infusion, auch in der Frühphase der Erkrankung eingesetzt, zu einer Verkürzung der Erkrankungsdauer und verbesserten Oxygenierung führen könnte [24, 25].

\section{Zusammenfassung}

Die besonderen Herausforderungen, die SARS-CoV-2 als Erreger dieser Pandemie an die Gesellschaft im Allgemeinen, aber auch an die Infektiologie stellt, sind enorm und vielfältig. Die Infektiosität von asymptomatischen Trägern stellt eine besondere Herausforderung für die Kontrolle der Pandemie dar. Zudem ist abschließend zu bemerken, dass wir bisher keinen aktiven Impfstoff gegen die Familie der Coronaviren vorzuweisen haben.

Die intensive interdisziplinäre Zusammenarbeit auf allen Ebenen weist hier allerdings bereits Erfolg versprechende erste Resultate auf.

\section{Offene Fragen}

Trotz der Fülle an Veröffentlichungen über COVID-19 bestehen sehr viele offene Fragen, die für die Infektiologie dringliche Relevanz haben:

- Mutationen: Bisher fehlen eindeutige Hinweise auf eine wesentliche, klinisch relevante genetische Veränderung von SARS-CoV-2. Engmaschige phylogenetische Analysen hierzu könnten wichtig für die weitere Epidemiologie und das Design von Impfstoffen sein.

- Diagnostik: Wie weit sind wir in der Entwicklung von Schnelltests, die mit hoher Sensitivität und Spezifität patientennah und auch im Feld eingesetzt werden können?

- Immunität: Welches sind die Korrelate einer protektiven Immunität? Welche Charakteristiken weisen diese in Patienten mit schweren, aber auch leichten Verläufen auf? Wie lange besteht diese Immunität und welche Rolle spielt eine potenzielle Kreuzimmunität zu anderen Coronaviren in der Pathogenese von COVID-19?

- Prolongierte SARS-CoV-2-PCR-Positivität: Welche Daten werden ausreichen, um die Dauer der Infektiosität und somit notwendiger Isolation besser zu definieren?

- Biomarker: Welche Biomarker werden sich zur Stratifizierung und Behandlung von Patientenkohorten etablieren?

- Langzeitprognose: Was ist die Pathophysiologie und v. a. die Prognose von Patienten mit persistierender Symptomatik nach durchgemachter Infektion?

\section{KERNAUSSAGEN}

- Die Behandlung von Patienten mit COVID-19 erfordert ein hohes Maß an interdisziplinärer Zusammenarbeit auf allen Ebenen.

- Eine besondere Herausforderung stellt die Infektiosität von infizierten Personen vor dem Auftreten von Symptomen dar.

- Der Krankheitsverlauf kann biphasisch verlaufen mit einer initialen Phase der viralen Replikation mit meist milder bis moderater Symptomatik. Dies kann gefolgt sein von einer klinisch schwer verlaufenden inflammatorischen Phase, ausgelöst durch eine überschießende Immunreaktion.

- Zu den therapeutischen Ansätzen gehören Substanzen wie Remdesivir, Tocilizumab sowie die Gabe von Rekonvaleszentenplasma.

- Die Frage der Immunität nach abgelaufener Infektion ist ebenso wie viele andere Aspekte dieser neuartigen viralen Infektion Gegenstand laufender Untersuchungen.

Interessenkonflikt 


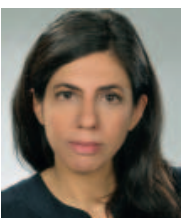

\section{Dr. med. Elham Khatamzas, PhD}

ist Funktionsoberärztin an der Medizinischen Klinik III des Klinikums der Ludwigs-MaximiliansUniversität München.

elham.khatamzas@med.uni-muenchen.de

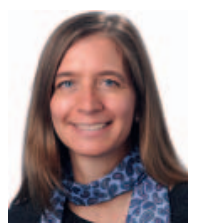

\section{Dr. med. Camilla Rothe}

ist Leiterin der Ambulanz für Tropen- und Reisemedizin an der Abteilung für Infektionsund Tropenmedizin des Klinikums der LudwigMaximilians-Universität München.

rothe@Irz.uni-muenchen.de

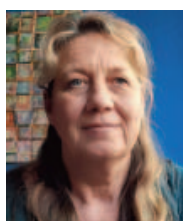

\section{PD Dr. med. Inge Kroidl}

ist Leiterin der Arbeitsgruppe „Vernachlässigte Tropenerkrankungen, Schwerpunkt Helminthen " an der Abteilung für Infektions- und Tropenmedizin des Klinikums der LudwigMaximilians-Universität München. ikroidl@Irz.uni-muenchen.de

\section{Korrespondenzadresse}

\section{Dr. med. Elham Khatamzas, PhD}

Medizinische Klinik III, Klinikum der Ludwigs-MaximiliansUniversität München

elham.khatamzas@med.uni-muenchen.de

\section{Literatur}

[1] Zhou P, Yang XL, Wang XG et al. A pneumonia outbreak associated with a new coronavirus of probable bat origin. Nature 2020; 579: 270-273

[2] Andersen KG, Rambaut A, Lipkin WI et al. The proximal origin of SARS-CoV-2. Nat Med 2020; 26: 450-452

[3] Hoffmann M, Kleine-Weber H, Schroeder S et al. SARS-CoV-2 Cell Entry Depends on ACE2 and TMPRSS2 and Is Blocked by a Clinically Proven Article SARS-CoV-2 Cell Entry Depends on ACE2 and TMPRSS2 and Is Blocked by a Clinically Proven Protease Inhibitor. Cell 2020; 181 (2): 271-280

[4] He X, Lau EHY, Wu P et al. Temporal dynamics in viral shedding and transmissibility of COVID-19. Nat Med 2020; 26: 672-675

[5] Rothe C, Schunk M, Sothmann P et al. Transmission of 2019NCOV infection from an asymptomatic contact in Germany. N Engl J Med 2020; 382: 970-971

[6] Liu Y, Ning Z, Chen Y et al. Aerodynamic analysis of SARS-CoV-2 in two Wuhan hospitals. Nature 2020. doi:10.1038/s41586 020-2271-3. Online ahead of print

[7] van Doremalen N, Bushmaker T, Morris D et al. Aerosol and Surface Stability of SARS-CoV-2 as Compared With SARS-CoV-1. N Engl J Med 2020; 382: 1564-1567. doi:10.1056/NEJMc2004973

[8] Zhou F, Yu T, Du R et al. Clinical course and risk factors for mortality of adult inpatients with COVID-19 in Wuhan, China: a retrospective cohort study. Lancet 2020; 395: 1054-1062

[9] Tay MZ, Poh CM, Rénia L et al. The trinity of COVID-19: immunity, inflammation and intervention. Nat Rev Immunol 2020; 20: $363-374$
[10] Huber TB, Glatzel M, Wichmann D et al. Correspondence Multiorgan and Renal Tropism of SARS-CoV-2. NEJM May 2020. doi:10.1056/NEJMc2011400

[11] Li ], Zhang L, Liu B et al. Case Report: Viral Shedding for 60 Days in a Woman with Novel Coronavirus Disease (COVID-19). Am J Trop Med Hyg 2020; 102: 1210-1213

[12] Wölfel R, Corman VM, Guggemos W et al. Virological assessment of hospitalized patients with COVID-2019. Nature 2020 581: 465-469

[13] Lin WHW, Kouyos RD, Adams RJ et al. Prolonged persistence of measles virus RNA is characteristic of primary infection dynamics. Proc Natl Acad Sci U S A 2012; 109: 14989-14994

[14] Radon K, Saathoff E, Pritsch M et al. Protocol of a populationbased prospective COVID-19 cohort study Munich, Germany (KoCo19). medRxiv 2020. doi:2020.04.28.20082743

[15] Streeck H, Schulte B, Kuemmerer B et al. Infection fatality rate of SARS-CoV-2 infection in a German community with a super-spreading event. medRxiv 2020 doi:2020.05.04.20090076

[16] Long QX, Liu BZ, Deng HJ et al. Antibody responses to SARSCoV-2 in patients with COVID-19. Nat Med 2020; 26 (6): 845848

[17] Koehler P, Cornely OA, Böttiger BW et al. Shimabukuro-Vornhagen A. COVID-19 Associated Pulmonary Aspergillosis. Mycoses 2020; 63 (6): 528-534

[18] Lee EYP, Ng MY, Khong PL. COVID-19 pneumonia: what has CT taught us? Lancet Infect Dis 2020; 20: 384-385

[19] Vogel-Claussen J, Ley-Zaporozhan J, Agarwal P et al. Recommendations of the Thoracic Imaging Section of the German Radiological Society for clinical application of chest imaging and structured CT reporting in the COVID-19 pandemic. Rofo 2020. doi:10.1055/a-1174-8378. Online ahead of print

[20] Rawson T, Morre L, Zhu N et al. Bacterial and Fungal co-infection with coronavirus: A rapid review to support COVID-19 antimicrobial prescribing. Clin Infect Dis 2020. doi:10.1093/ cid/ciaa530. Online ahead of print

[21] Mehra MR, Desai SS, Ruschitzka F et al. Hydroxychloroquine or chloroquine with or without a macrolide for treatment of COVID-19: a multinational registry analysis. Lancet 2020. doi:10.1016/S0140-6736(20)31180-6. Online ahead of print

[22] Watson ]. An open letter to Mehra et al and The Lancet. Im Internet: https://zenodo.org/record/3862789\#.XwW4vygzaUl; Stand: 29.05.2020

[23] Mulangu S, Dodd LE, Davey RT et al. A randomized, controlled trial of Ebola virus disease therapeutics. N Engl J Med 2019; 381: 2293-2303

[24] Shen C, Wang Z, Zhao F et al. Treatment of 5 Critically III Patients with COVID-19 with Convalescent Plasma. JAMA - J Am Med Assoc 2020; 323: 1582-1589

[25] Duan K, Liu B, Li C et al. Effectiveness of convalescent plasma therapy in severe COVID-19 patients. Proc Natl Acad Sci U S A 2020; 117: 9490-9496

\section{Bibliografie}

DOI https://doi.org/10.1055/a-1164-3960

Dtsch Med Wochenschr 2020; 145: 1051-1056

(C) Georg Thieme Verlag KG, Stuttgart · New York ISSN 0012-0472 